\title{
Agranuläre CD4/CD56 positive hämato-dermale Neoplasie (CD56 positives Non-Hodgkin-Lymphom)
}

\author{
Agranular CD4/CD56 Positive Hematodermic Neoplasm \\ (CD56 Positive Non-Hodgkin Lymphoma)
}

Autoren

Institut

\section{S. Halm, P. von den Driesch}

Klinik für Dermatologie und Allergologie, Zentrum für Hautkrankheiten, Klinikum Stuttgart

\section{Bibliografie}

DOI $10.1055 / \mathrm{s}-2007-995759$

Akt Dermatol 2008; 34 :

192-195 @ Georg Thieme

Verlag KG Stuttgart · New York ISSN 0340-2541

Korrespondenzadresse Prof. Dr. Peter von den Driesch Klinik für Dermatologie und Allergologie

Zentrum für Hautkrankheiten

Klinikum Stuttgart

Prießnitzweg 24

70374 Stuttgart

pdriesch@kbc-intern.de

\section{Zusammenfassung \\ $\nabla$}

Die CD4+/CD56+ hämatodermale Neoplasie ist ein seltenes primär kutanes Lymphom und bisher einziger Vertreter der Klasse der hämatologischen Vorläuferneoplasien. Die Tumorzellen exprimieren CD56 und wurden deshalb lange als Natural-Killer(NK)-Lymphom angesehen. Da aber regelmäßig auch CD123 Expression gefun-

\section{Einleitung}

$\nabla$

Zu den primär kutanen Lymphomen, die zu der Gruppe der extranodalen Non-Hodgkin-Lymphome zählen, gehören neben den mit ca $70 \%$ häufigsten kutanen T-Zell Lymphomen und den mit ca. 25\% vertretenen kutanen B-Zell Lymphomen auch die hämatologischen Vorläuferneoplasien. Einziger Vertreter dieser Klasse ist die CD56+ CD123 + hämatodermale Neoplasie (HN). Wir zeigen in dieser Kasuistik einen solchen Fall.

\section{Kasuistik \\ $\nabla$}

\section{Anamnese}

Aufnahme eines 80-jährigen dementen adipösen Patienten in gutem AZ mit vorbekanntem Diabetes mellitus Typ II (mit Polyneuropathie, Retinopathie, Katarakt), COPD und Silikose, arterieller Hypertonie, kompensierter Herzinsuffizienz, chronischer kompensierter Niereninsuffizienz, BPH und Hyperurikämie.

Der Patient gab an, seit ca. 8 Wochen unter pruriginösen Plaques am gesamten Rumpf und im Gesicht zu leiden. Im Pflegeheim sei keine Lokaltherapie erfolgt, nach einer Vorstellung beim Hautarzt kam es zunächst zur ambulanten Vorstellung bei uns und daraufhin zur stationären Aufnahme. den wurde, gilt der Ursprung aus prädendritischen Zellen als gesichert. Der Tumor weist einen meist aggressiven klinischen Verlauf mit hoher Mortalitätsrate durch Lymphknoten und Knochenmarksbefall mit folgender leukämischer Phase auf. Wir stellen einen Patienten vor, bei dem eine systemische Chemotherapie nach dem KNOSPE-Schema bisher einen zufriedenstellenden Verlauf erzielte.

\section{Dermatologischer und körperlicher Aufnahmebefund}

Am gesamten Integument sowie an der Stirn zeigten sich disseminierte, scharf begrenzte, nummuläre, teilweise derb infiltrierte, livide Plaques von bis zu $4 \mathrm{~cm}$ Durchmesser ( Abb.1a-d). Axillär beidseits waren derb vergrößerte, nicht schmerzhafte Lymphknoten palpabel. Die weiteren Lymphknoten zervikal und inguinal beidseits stellten sich nicht vergrößert dar. Nebenbefundlich bestanden Unterschenkelödeme beidseits mit beginnender Stauungsdermatitis und ein Druckulkus am rechten Fußrücken.

\section{Histologische Befunde}

Hist. J. Nr. 5432/07: Unter einer unauffälligen Epidermis zeigen sich im gesamten Corium kompakte Infiltrate aus großen lymphoiden Zellen mit pleomorphen Zellkernen und atypischen Mitosen ( $\bullet$ Abb. 3a). Die atypischen Infiltrate dringen deutlich in das subkutane Fettgewebe ein, hier ausgeprägtes „Rimming“ ( $\bullet$ Abb. 3 b). Immunhistologisch sind die Zellen positiv für CD56 ( $\bullet$ Abb. 3c) und CD4, hingegen überwiegend negativ für den Pan-T-Zellmarker und den Pan-B-Zellmarker sowie CD8. Die Proliferation mit Ki67 ist hoch. Nur einzelne Blasten exprimieren das Ki1 Antigen. 

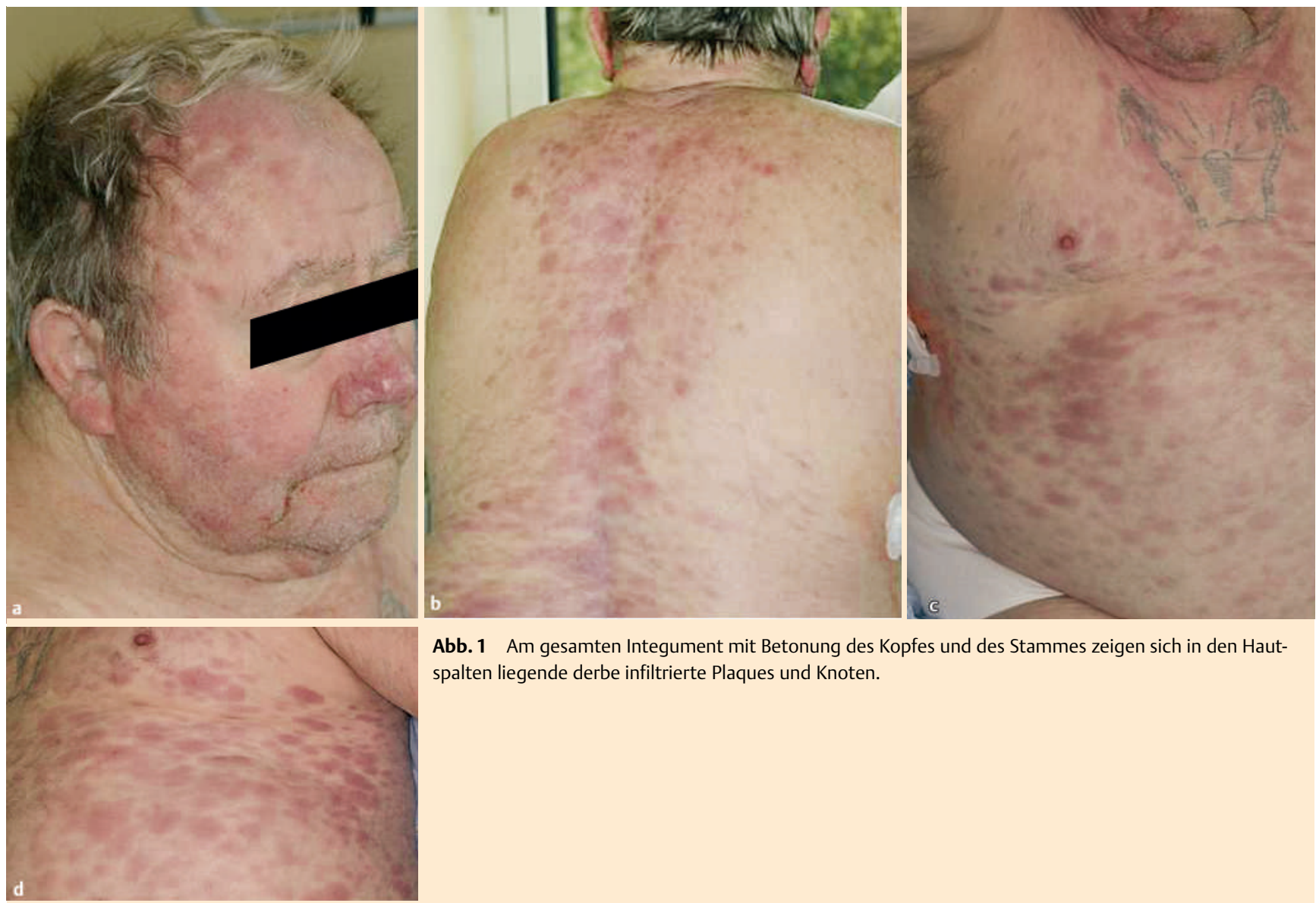

Abb. 1 Am gesamten Integument mit Betonung des Kopfes und des Stammes zeigen sich in den Hautspalten liegende derbe infiltrierte Plaques und Knoten.

Passt zu einer Infiltration der Haut durch ein CD56 positives sog. NK-Lymphom. Hiervon rein (immun)histologisch nicht abzugrenzen ist eine CD56 positive hämatodermale Neoplasie.

\section{Pathologisch anatomische Begutachtung durch das Referenzzentrum für Lymphknotendiagnostik und Hämatopathologie Prof. Dr. Feller in Lübeck:}

Histologie: relativ dichtes, angedeutet bandartiges in der Tiefe auch fleckförmiges lymphoidzelliges Infiltrat. Relativ monomorphe Population aus intermediär großen Zellen mit irregulär geformten hellen Kernen und kleinen Nukleolen. Das Zytoplasma weit und hell. Immer wieder eine Mitose. Einzeln eingestreute reife Mastzellen. Kein Nachweis PAS-positiver Pilzhyphen oder -sporen.

Immunhistochemie: Positivität für CD56, CD4, Negativität gegenüber CD8, CD2, CD5, CD34, KP1. Keine Expression von CD3, kein vermehrter Nachweis von TIA 1 oder CD30. Nur wenige eingestreute CD20 positive B-Zellen. An den MiB 1 positiven Zellen gemessene erhöhte Proliferationsrate von 50\%.

Molekulargenetische Analyse/Klonalitätsanalyse (TCR): In der Untersuchung des T-Zell-Rezeptor-Gamma-Ketten-Gen-Locus zeigte sich ein reproduzierbarer monoklonaler Peak in polyklonalem Hintergrund in der MIXB-PCR.

In der Zusammenfassung passt der Befund zu einer Infiltration der Haut durch ein T-NK-Zell-Lymphom.

Knochenmarkshistologie: Der histologische KM-Befund entspricht einer hochgradigen subtotalen Infiltration des Knochenmarks durch das bekannte Lymphom mit Verdrängung der Hämatopoese.
Knochenmarkszytologie: Der zytologische KM-Befund ist schwierig zuordenbar, am ehesten handelt es sich um eine subtotale Infiltration durch eine hämatologische Neoplasie, am ehesten durch ein Lymphom niedrigen Malignitätsgrades mit Verdrängung der Hämatopoese.

Labor bei Aufnahme

Routine Labor:

(Diff-)Blutbild: Hb 12,9 g/dl, Hkt 37,5\%, Erys 4,29 Mio/ $\mu \mathrm{l}, 33 \%$ Segmentkernige, 11\% Monozyten, 10\% Stabkernige Granulozyten, $4 \%$ Metamyelozyten

klin. Chemie: LDH $400 \mathrm{U} / \mathrm{l}$, Krea $1,6 \mathrm{mg} / \mathrm{dl}$, Hs $12 \mathrm{mg} / \mathrm{dl}$, HST $38 \mathrm{mg} / \mathrm{dl}$.

\section{Lymphozytentypisierung/FACS Analyse aus peripherem} Blut:

erhöht - CD4/CD8 Ratio aufgrund prozentual verminderter zytotox. T-Zellen. Atypische Zellpopulation (15\% aller kernhaltigen Zellen im Lymphozytenfenster) mit Expression von HLA-DR+, CD56+, CD4+, CD117+ und fraglich CD33.

\section{Apparative Diagnostik}

Röntgen Thorax: grenzwertig großes Herz in seitlicher Projektion, Hili beidseits kräftig, jedoch gefäßartig auffasernd. Das obere Mediastinum mäßig verbreitert. V.a. Struma ohne Trachealpelottierung. Keine Stauungszeichen oder Pleuraerguss, keine Infiltrate. Leichter dorsaler Zwerchfellhochstand links. Spondylosis deformans und vordere Längsbandverkalkung der BWS. 


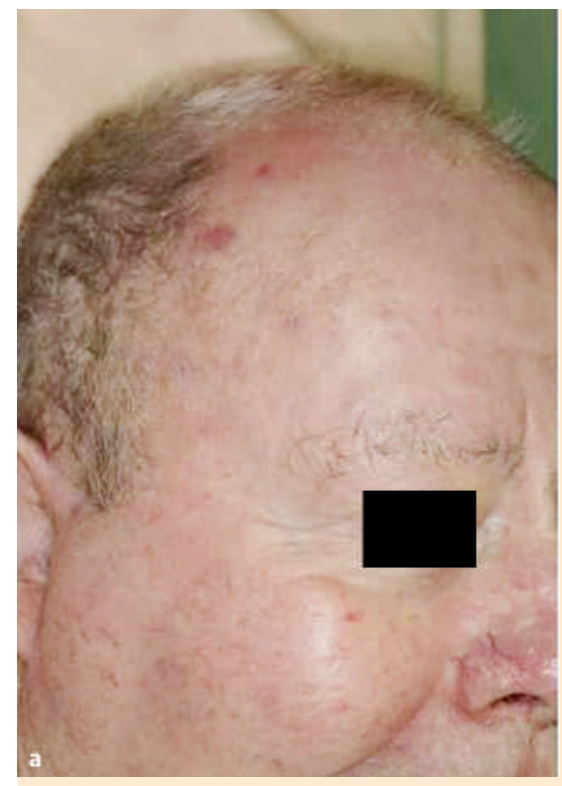

Abb. 2 Deutliche Remission nach zwei Zyklen Knospe-Schema.

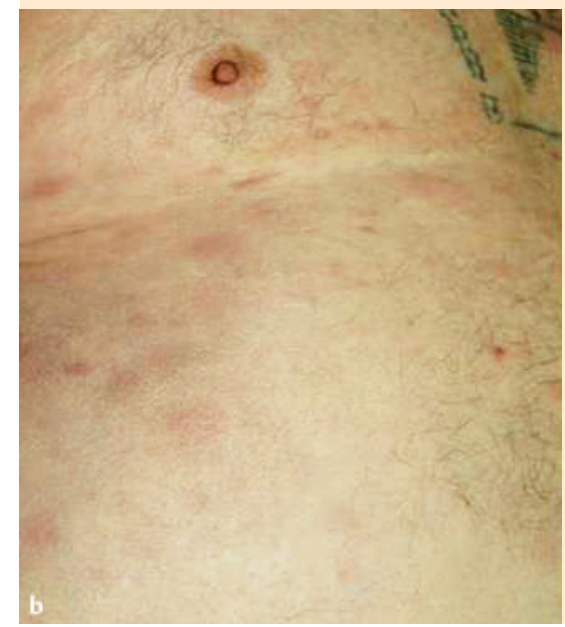

CT Thorax und Abdomen nativ: Es finden sich axillär bds. sowie vor allem inguinal bds. vergrößerte suspekte Lymphknotenpakete, paraaortal und parailiacal keine suspekten Lymphknoten.

\section{Therapie und Verlauf}

$\nabla$

Während des stationären Aufenthaltes bei uns erfolgte die top. Therapie des Integuments mit initial Betamethason $0,1 \%$ in Ungt. Leniens, bei Entlassung Triamcinolon 0,1\% in Ungt. leniens jeweils in Kombination mit Chinosol 2\% in Ungt. leniens.

Zusätzlich begannen wir nach einer augenärztlichen Kontrolluntersuchung mit einer systemischen PUVA (Mo-Fr) mit Gabe von 5 Tabletten Meladinine ${ }^{\circledR} 2$ Stunden vor UV-Exposition.

Zur Therapie der Stauungsdermatitis wurden Kompressionsverbände nach Pütter angelegt, das Druckulkus behandelten wir mit Hydrokolloidverbänden.

Unter dieser Therapie zeigten sich die oben beschriebenen Hautveränderungen nur minimal rückläufig.

Nach Diagnosestellung und hämatologischer Rücksprache wurde die systemische palliative Chemotherapie nach dem KNOSPESchema eingeleitet:

Chlorambucil 0,7 mg/kg Körpergewicht verteilt über 3 Tage p. o.

Prednisolon $75 \mathrm{mg}$ Tag 1,50 mg Tag 2, $25 \mathrm{mg}$ Tag 3 p. o.
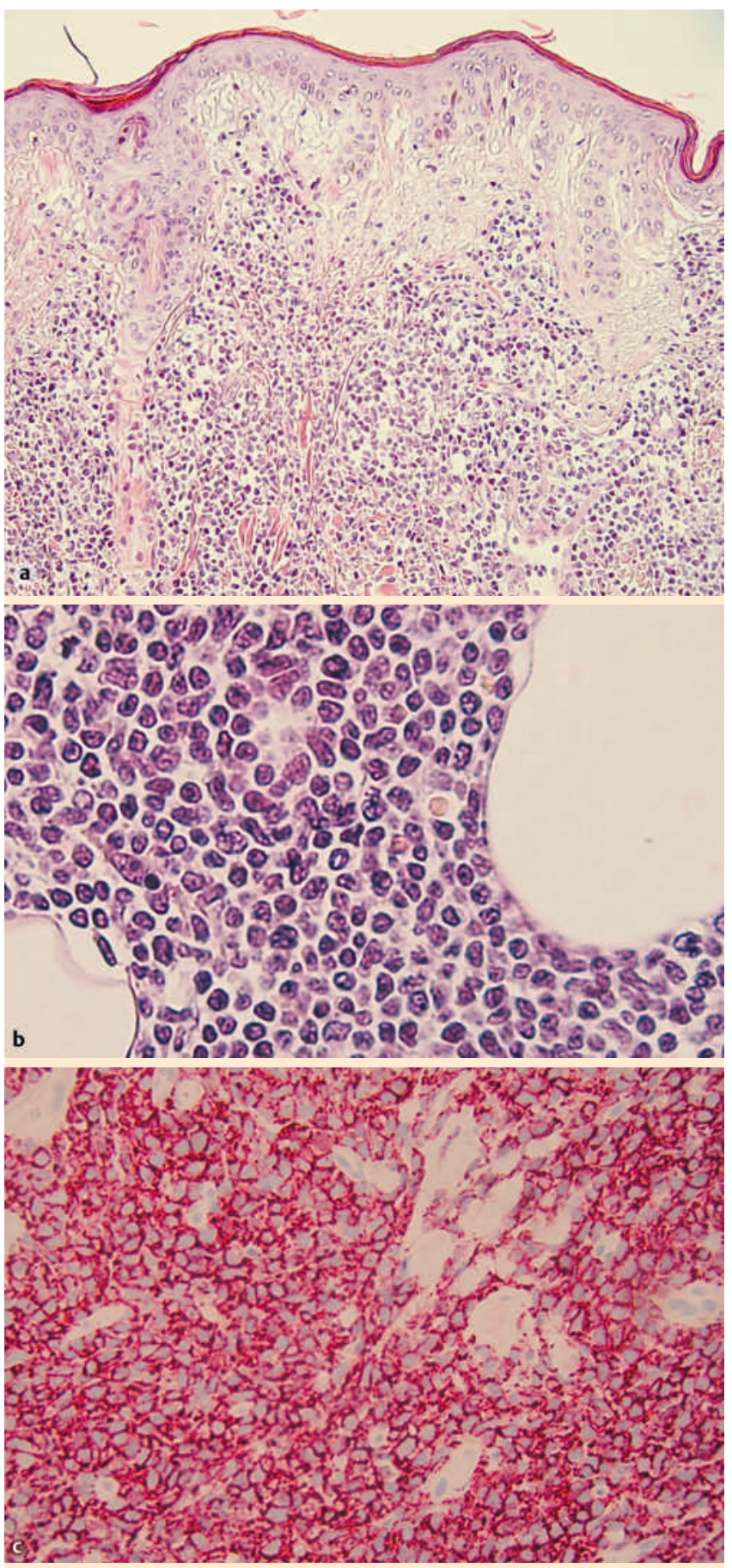

Abb. 3 a Kompakte Infiltrate aus atypischen lymphoiden Tumorzellen und deutlicher Beteiligung des Fettgewebes (b) und CD56 Expression (c).

Die Empfehlung der Onkologen war, zunächst 8-10 Zyklen durchzuführen.

Nach zwei Zyklen zeigten sich die Hautveränderungen deutlich rückläufig ( Abb.2a,b). Trotz umfangreicher Aufklärung des Patienten und seiner Angehörigen wurde eine weitere Therapie abgelehnt. 


\section{Diskussion}

$\nabla$

Aufgrund des klinischen Bildes kam zunächst das ganze Spektrum der kutanen Lymphome in Betracht. Nach Eingang des Differenzialblutbildes zeigte sich rasch, dass es sich um eine hämatologische Systemerkrankung handelte, dies bestätigte sich dann in der Bildgebung sowie der Knochenmarksdiagnostik und der FACS-Analyse. Entscheidend für die endgültige Diagnosefindung war letztendlich die Histologie mit der typischen Einbeziehung des subkutanen Fettgewebes sowie die Immunhistologie. Wie in der Literatur beschrieben, handelt es sich bei der CD4+/CD56+ hämatodermalen Neoplasie um eine seltene Leukämie CD123+ prädendritischer Zellen mit typischer rascher Remission der Hautveränderungen unter initialer Chemotherapie, die in Diskrepanz steht zu der schlechten Prognose bezüglich des Gesamtüberlebens. Dies wird mit 12-14 Monaten angegeben. Typisch ist eine regelhaft therapeutisch schlecht zu beeinflussende terminale leukämische Phase.

Bei unserem Patienten zeigten sich unter der milden Chemotherapie die kutanen Infiltrate erwartungsgemäß rasch rückläufig, ebenso der Puritus. Dennoch ist die Prognose quoad vitam mit Vorsicht zu stellen.

\section{Abstract}

\section{Agranular CD4/CD56 Positive Hematodermic Neoplasm (CD56 Positive Non-Hodgkin Lymphoma) $\nabla$}

CD4+/CD56+ hematodermic neoplasm is a rare type of primary cutaneous T-cell lymphoma and so far the single representative of the category precursor hematologic neoplasm. The tumor cells express CD56 and have therefore been suggested to represent a natural-killer(NK)-cell-lymphoma. The finding of CD123 positivity on the tumour cells now assures the origin from plasmacytoid dendritic cells. The tumour mostly shows a clinical course with high mortality caused by lymph node and bone marrow involvement often followed by a terminal leukemic phase. We present a patient treated with systemic chemotherapy (KNOSPE-scheme) and satisfactory results until now.

\section{Literatur}

1 Dummer R, Cozzio A, Urosevic M. Pathogenesis and therapy of cutaneous lymphomas - Progress or impasse? Exp Dermatol 2006; 15: 392 400

2 Dummer R, Stadler R, Sterry W. Cutaneous lymphomas. JDDG 2007; 5: 605-617

3 Petrella T, Bagot M, Willemze R. Blastic N-Cell Lymphomas. Am J Clin Pathol 2005; 123: 662 - 675

4 Stadler R. Kutane Lymphome. Hautarzt 2006; 57: $744-755$

5 Willemze R, Jaffe ES, Burg G. WHO EORTC classification for cutaneous lymphomas. Blood 2005; 105: 3768 - 3785

6 Dummer R, Asagoe K, Cozzio A. Recent advances in cutaneous lymphomas. Journal of Dermatological Science 2007; 48: 157 - 167

7 Petrella T, Comeau MR, Maynadie M. Agranular CD4+/CD56+ hematodermic neoplasm originates from a population of CD56+ precursor cells related to plasmacytoid monocytes. Am J Surg Pathol 2002 Jul; 26: $852-862$

8 Niakosari F, Sur M. Agranular CD4+/CD56+ Hematodermic Neoplasm: A distinct Entity described in the Recent WHO-EORTC Classification for cutaneous lymphomas. Archives of Pathology and Laboratory Medicine 2007; 131: 149-151 